\title{
Reseña
}

\section{El reto inter y transdisciplinar de la escuela en la sociedad actual ${ }^{1}$}

Francisco García ${ }^{2}$

Universidad Distrital Francisco José de Caldas

Bogotá, Colombia

Barreneche, C., Cabra, F., Hernández, I., Niño, R., Rodríguez, J., \& Marciales, G. (2018). Las ciencias sociales y humanas en la actual sociedad del conocimiento: Escenarios de indagación ínter y transdisciplinar. Editorial Pontificia Universidad Javeriana.

\section{${ }^{1}$ CÓMO CITAR ESTA RESEÑA}

García, F. (2021). El reto inter y transdisciplinar de la escuela en la sociedad actual. Lenguaje, 49(1), 226-230. https://doi.org/10.25100/lenguaje.v49i1.9856.

2 Docente, pedagogo y Magíster Universidad Distrital Francisco José de Caldas.

Correo electrónico: fagarciat@correo.udistrital.edu.co 
El texto Las ciencias sociales y humanas en la actual sociedad del conocimiento, escrito por Barreneche et al. (2018) es producto del colectivo de profesores pertenecientes al Doctorado en Ciencias Sociales y Humanas de la Pontificia Universidad Javeriana. En él se analiza los diferentes retos que se asocian al desarrollo de la sociedad de la información y la revolución digital en los diversos campos del conocimiento, incluyendo la educación, la cultura, la política, la comunicación y las nuevas visiones de humanismo.

El texto examina la forma en la cual las ciencias humanas deben establecer nuevos procesos de comprensión, transformando las perspectivas más tradicionales, que permitan no solo comprender el impacto de la evolución tecnológica, sino también las dinámicas asociadas a la articulación entre las redes electrónicas, el desarrollo social, las formas de habitabilidad, de comprensión y de relacionamiento entre las personas y las máquinas. En este sentido, los autores explican que la evolución crítica de la sociedad, proyectada hacia el futuro, depende de la investigación transdisciplinar que promueve nuevas perspectivas desde las ciencias sociales con diálogos entre lo humano, lo ecofilosófico y los procesos socioeducativos.

El libro, en su composición, se divide en tres apartados centrales. El primero corresponde a los dos primeros capítulos: "Educar en la Esperanza" y "Tecnologías cognitivas y educación emergente". En ellos, se analizan procesos educativos potenciados por el desarrollo de la tecnología digital y sus nuevos ecosistemas de interacción. El primer capítulo toma como marco de referencia la localidad de Soacha y los resultados del proyecto Smart Town, en el cual se utilizaron las tecnologías para generar procesos de comunicación y de apropiación local del territorio. Los autores plantean que avanzar en el camino definitivo hacia la paz depende del desarrollo de nuevas estrategias pedagógicas que ayuden a generar -en los jóvenes- maneras de construir conocimientos críticos y procesos de formación política; todo ello, orientando por una exploración del concepto de esperanza, sus posibilidades epistemológicas y su potencial para consolidar el diálogo, la verdad, la cultura política y la solidaridad.

En el segundo capítulo los autores analizan el concepto de sistema tecnosocial, desde el cual se comprende la incidencia de las tecnologías en la comunicación, en la afectividad y en el desarrollo de esquemas de comprensión colectivos a partir de entornos artificiales y abstractos. Se plantea cómo estos ecosistemas digitales establecen desafíos para la educación crítica y evidencian la configuración de subjetividades diversas y fragmentadas. Como conclusión, se identifica una educación emergente que debe dialogar con la complejidad y la incertidumbre.

A través de los dos primeros capítulos, un aspecto central es el análisis de una humanidad que permanece en un constante proceso de reconfiguración, que evoluciona a medida que las tecnologías cambian y se imponen nuevas maneras de configurar la representación del mundo en un entorno caracterizado centralmente por la mutabilidad.

La segunda parte del libro comprende los capítulos "El sujeto de la razón métrica" y "El giro posdigital: convergencias, balances críticos y vulnerabilidades de una era". Los 
autores brindan un análisis en torno a los mecanismos a través de los cuales se difunde, sistematiza, ordena y controla la información. El capítulo sobre "El sujeto de la razón métrica" hace referencia a un sujeto que hoy en día es medido, cuantificado, valorado, limitado y evaluado a través de una serie de procesos digitalizados que tienen como finalidad identificar y dirigir sus opiniones, gustos e inclinaciones a través de redes digitales, estableciendo lo que los autores denominan procesos de biocontrol. En conjunto, la información que se sistematiza a través de la red digital permite generar una serie de algoritmos mediante los cuales es posible configurar diferentes tipos de usuarios, así como tener un mayor control de las dinámicas sociales, facilitando de esta manera los procesos de vigilancia.

Por otro lado, en el capítulo que habla del giro posdigital se analiza la forma en que las personas comprenden la información y la producción discursiva, mediante procesos de construcción y difusión hipertextual, además del desarrollo de una tendencia hacia la hiperconexión. Existen en el apartado dos posiciones: aquellas que se centran en demostrar los avances que ha traído consigo la era posdigital; sirviendo como puente entre la bioinformación, las poéticas digitales, los lenguajes híbridos y los campos del conocimiento que evolucionan a la par de la innovación social y política que traen consigo las tecnologías emergentes.

También aquellas que resaltan las críticas según las cuales las dinámicas mercantiles o las acciones autoritarias del Estado limitan las posibilidades reales de conexión e interactividad mediadas por las tecnologías digitales. Sin embargo, los autores plantean que estas críticas están ligadas también a un reconocimiento de las potencialidades y posibilidades de las tecnologías, de tal manera que no se establecen como una reacción tecnofóbica sino desde la necesidad de adaptar la tecnología funcionalmente, según las necesidades sociales. El aporte de este apartado es la comprensión que hace de la sociedad de la información un espacio social que establece puentes entre el hombre, la cultura, el desarrollo tecnológico de software y las nuevas ecologías digitales.

En ambos capítulos se reconoce que en la ¿actualidad? se ha venido desarrollando una tendencia que consiste en utilizar la lectura e interpretación de los datos masivos como medio para establecer mecanismos de vigilancia de los individuos y una forma de comprensión del comportamiento de las comunidades. Esto es posibilitado por la baja comprensión de los usuarios sobre cómo se usan sus datos y cómo se lee su comportamiento en la red.

Finalmente, en la tercera parte se incluyen dos nuevos capítulos enfocados centralmente en el papel que desempeña la ecopolítica en medio de este proceso de trasformación digital. En el primer capítulo de este apartado: "Mundos posibles no lineales: ecopolítica", se desarrolla una manera de entender al hombre desde una perspectiva de la complejidad, es decir en el marco de un mundo cada vez más diverso e interconectado. Se evidencia, por tanto, un cambio de tendencia del antropocentrismo al 
alocentrismo, lo cual implica analizar una serie de cuestiones más amplias que obligan a pensar lo humano en relación con otros sistemas de vida artificiales, los cuales tienen una participación sumamente relevante en el desarrollo social y en la sostenibilidad del planeta.

En este capítulo los autores también asocian el concepto de mundos posibles con la necesidad de orientar el estudio sobre la ecopolítica asumiendo la complejidad en la organización del mundo actual, en donde se evidencia la divergencia de la vida social ante la existencia de nuevas formas de ocupar el mundo. De esta manera, los mundos posibles abren la perspectiva de un mundo interconectado y diverso, en el cual se establece un proceso de interacción entre la vida humana, la vida artificial y la biocomputación. Los autores explican que solo comprendiendo las diversas posibilidades en la construcción de los mundos posibles es posible orientar el desarrollo de una ecología que sea capaz de asumir la complejidad del mundo actual, caracterizada por una relación entre diversos campos del conocimiento, relaciones colectivas, desarrollos tecnológicos y cambios en los procesos educativos.

Finalmente, en el capítulo: "Ciencias sociales poshumanas: sociedades artificiales" se analizan los problemas asociados a un escenario poshumano, en el cual es necesario ampliar la concepción de subjetividad a otras formas vivientes, como las plantas y los animales, pero también en nuevos ecosistemas dominados por la vida artificial. En este sentido, la ecopolítica permite comprender la vida como un sistema que permanece en un constante proceso de trasformación, lo cual obliga a pensar en la inevitabilidad de los procesos de reconfiguración humana como clave central y como consecuencia también de los procesos de renovación del conocimiento, que en un futuro no precisarían de una subjetividad ligada a lo humano sino al conjunto de relaciones que se establecen con lo bioinformacional.

En este sentido, los autores observan que la incidencia de las tecnologías en la sociedad no solo pone en evidencia una serie de transformaciones y reconfiguraciones en la vida humana, sino que también ayuda a comprender los límites de lo ético y de lo estético en relación con la transformación de las prácticas sociales y de la investigación científica; ya que el sujeto y los medios que tiene para interpretar la realidad permanecen siempre en un proceso de reconfiguración.

Los textos que componen este apartado final tienen como base la interrelación de los diferentes sistemas (humanos, tecnológicos, computacionales y ecológicos), como elemento central en el desarrollo de estrategias que promuevan trasformaciones relevantes y acertadas que orienten las dinámicas de desarrollo social. Aunque los textos plantean escenarios investigativos y recuperan tendencias teóricas no todos incorporan análisis de casos, lo cual permitiría comprender mucho mejor las ideas propuestas desde una perspectiva más práctica.

Este libro es pertinente en el contexto actual, en la medida en que ofrece un análisis transversal en relación con el desarrollo tecnológico y la forma en que afecta cada uno de 
los procesos mediante los cuales se vive, se piensa, se siente, se educa y se aprende. Introduce conceptos que permiten pensar y nombrar nuevas realidades y dinámicas de interacción, desde una perspectiva transdiciplinaria. analiza con profundidad los nuevos retos a los cuales se enfrentan las sociedades en una época caracterizada por el desarrollo de la interconexión digital como principal elemento de transformación y se centra en la construcción de estructuras abiertas y dinámicas para explicar lo humano. 